\title{
Magnetic Resonance Spectroscopy
}

National Cancer Institute

\section{Source}

National Cancer Institute. Magnetic Resonance Spectroscopy. NCI Thesaurus. Code C16810.

Detection and measurement of the resonant spectra of molecular species in a tissue or sample. 